\title{
Tipos de violência no trabalho da enfermagem na Estratégia Saúde da Família
}

\author{
Types of workplace violence in nursing in the Family Health Strategy \\ Tipos de violencia en el trabajo de enfermería en la Estrategia de Salud Familiar
}

Grasiele Fatima Busnello ${ }^{1,2}$ (1)

Letícia de Lima Trindade ${ }^{1,2}$ (D)

Daiane Dal Pai ${ }^{3}$ (b)

Carmem Lúcia Colomé Beck ${ }^{4}$ (1)

Olga Maria Pimenta Lopes Ribeiro 5 []

1. Universidade Comunitária da Região de

Chapecó. Chapecó, SC, Brasil.

2. Universidade do Estado de Santa Catarina.

Chapecó, SC, Brasil.

3. Universidade Federal do Rio Grande do Sul. Porto Alegre, RS, Brasil.

4. Universidade Federal de Santa Maria. Santa Maria, RS, Brasil.

5. Escola Superior de Enfermagem do Porto. Porto, Portugal.

\section{REsUMO}

Objetivo: analisar a ocorrência dos diferentes tipos de violência no trabalho da Enfermagem na Estratégia Saúde da Família e as implicações dos aspectos laborais e do trabalhador. Método: estudo misto, explanatório, sequencial, com 169 trabalhadores de Enfermagem da Estratégia Saúde da Família. Como instrumentos de coleta de dados, foram utilizados o Survey Questionnaire Workplace Violence in the Health Sector e a entrevista semiestruturada. Resultados: foram encontrados episódios de agressão verbal com melhores médias na avaliação sobre o reconhecimento e os relacionamentos no trabalho e o maior uso de medicamentos. $\mathrm{O}$ assédio moral foi associado à cor da pele branca dos participantes, ao cargo de enfermeiro; apresentaram-se avaliações mais negativas acerca do reconhecimento laboral e dos relacionamentos interpessoais e uma maior preocupação com a violência. Já o assédio sexual relacionou-se ao cargo de técnico/auxiliar de Enfermagem e pela ausência de estímulo para os relatos de violência. A discriminação racial foi associada à cor da pele parda/negra e à redução da satisfação laboral. Conclusão e implicações para a prática: a análise dos fatores que se associam aos tipos específicos de violência permite melhor determinar medidas e políticas institucionais que minimizem os atos violentos contra os trabalhadores de Enfermagem.

Palavras-chave: Violência no Trabalho; Enfermagem; Saúde do Trabalhador; Estratégia Saúde da Família; Atenção Primária à Saúde.

\section{Abstract}

Objective: to analyze the occurrence of different types of violence in the work of Nursing in the Family Health Strategy and the implications of the labor and worker aspects. Method: a mixed, explanatory, sequential study, with 169 nursing workers of the Family Health Strategy. As instruments of data collection, the Survey Questionnaire Workplace Violence in the Health Sector and the semi-structured interview were used. Results: episodes of verbal aggression with better averages were found in the assessment of recognition and relationships at work and the highest use of medication. Workplace bullying was associated with the participants' white skin color, the position of nurse, more negative evaluations about work recognition and interpersona relationships, and a greater concern with violence. Sexual harassment was associated with the position of nursing technician/ auxiliary and the lack of encouragement to report violence. Racial discrimination was associated with brown/black skin color and reduced job satisfaction. Conclusion and implications for practice: the analysis of the factors that are associated with specific types of violence makes it possible to better determine institutional measures and policies that minimize violent acts against nursing workers.

Keywords: Violence at Work; Nursing; Worker Health; Family Health Strategy; Primary Health Care.

\section{REsumen}

Objetivo: analizar la ocurrencia de los diferentes tipos de violencia en el trabajo de enfermería en la Estrategia Salud de la Familia y las implicaciones de los aspectos laborales y trabajador. Método: estudio explicativo secuencial mixto con 169 trabajadores de enfermería de la Estrategia Salud de la Familia. Como instrumentos de recolección de datos se utilizó Cuestionario de Encuesta de Violencia Laboral en el Sector Salud e la entrevista semiestructurada. Resultados: se encontraron episodios de agresión verbal con mejores promedios en la evaluación del reconocimiento y las relaciones en el trabajo y mayor uso de medicamentos. El acoso moral se asoció con el color de piel blanco de los participantes, como enfermeras, presentaron valoraciones más negativas sobre el reconocimiento laboral y las relaciones interpersonales y mayor preocupación por la violencia. El acoso sexual, por su parte, se relacionó con el puesto de técnico / auxiliar de enfermería y la falta de estímulo para denuncias de violencia. La discriminación racial se asoció con el color de piel marrón / negro y una menor satisfacción laboral. Conclusión e implicaciones para la práctica: el análisis de los factores que se asocian a tipos específicos de violencia permite determinar mejor las medidas y políticas institucionales que minimicen actos violentos contra trabajadores de enfermería.

Palabras clave: Violencia en el Trabajo; Enfermería; Salud del Trabajador; Estrategia de Salud Familiar; Atención Primaria de Salud.
Autor correspondente:

Grasiele Fatima Busnello.

E-mail: grasibusnello@gmail.com.

Recebido em: 27/10/2020.

Aprovado em: 15/02/2021. 


\section{INTRODUÇÃO}

A violência no trabalho é considerada um fenômeno crescente, muitas vezes, naturalizado no cotidiano dos serviços brasileiros, raramente mensurado, tipificado ou compreendido, considerado um risco inevitável para alguns trabalhadores, porém, pouco combatido, tratado ou prevenido. No entanto, é passível de prevenção e constitui importante causa de lesão física e, principalmente, de adoecimento mental. ${ }^{1}$

A Organização Mundial da Saúde (OMS) define a violência como o "uso intencional ou ameaça de uso da força física contra outra pessoa ou si próprio que resulta, ou tem a probabilidade de resultar em lesão, morte, dano psicológico."2:5

O setor de saúde posiciona-se entre aqueles que denotam alto potencial para a ocorrência de agressões aos trabalhadores. Os profissionais da saúde estão expostos a diversos riscos, em grande parte, associados ao contato constante com o público. No que se refere à violência, $69 \%$ dos casos constituem-se em episódios envolvendo pacientes. ${ }^{3}$

Alguns estudos ${ }^{4,5}$ destacaram que a violência nos serviços de saúde atinge, principalmente, os trabalhadores que ficam responsáveis pela primeira comunicação direta com pacientes e seus familiares, ou seja, os profissionais de Enfermagem, os tornando mais vulneráveis. As pesquisas em outros países sinalizaram que, dentre os profissionais de saúde, a maior prevalência de episódios de violência envolve enfermeiros. ${ }^{4,6,7}$ A experiência frequente da exposição contribui para a gravidade da problemática, uma vez que os trabalhadores são vítimas frequentes desse fenômeno, potencialmente na Estratégia Saúde da Família (ESF). 8,9

Dentre os cenários de saúde no Brasil, destaca-se a ESF, principal modelo assistencial da Atenção Primária à Saúde (APS) no país e importante serviço no contexto do Sistema Único de Saúde (SUS). A APS, orientada pela Política Nacional de Atenção Básica (PNAB), também reconhecida como Atenção Básica em Saúde (ABS), no país, tem aportando-se na ESF e foi planejada para atender às necessidades de saúde dos indivíduos em sua singularidade e complexidade, visando à assistência integral, ${ }^{10}$ bem como as coletividades.

Neste contexto, os profissionais de Enfermagem têm sido considerados como grupo vulnerável à violência no trabalho. ${ }^{5}$ $\mathrm{Na}$ ESF, identifica-se a elevada ocorrência de insultos $(44,9 \%)$, ameaças $(24,8 \%)$ e agressão física $(2,3 \%)$, especialmente contra médicos e enfermeiros, estando estes profissionais mais propensos a manifestar sintomas depressivos e a depressão. ${ }^{11}$

$\mathrm{Na}$ busca por produções científicas, foram encontrados escassos estudos nacionais ${ }^{12,13} \mathrm{e}$ internacionais ${ }^{7,8}$ sobre a temática violência no trabalho da Enfermagem na ESF. Pressupõe-se, com isso, que exista uma lacuna a ser pesquisada, reafirmando a relevância de investigações na temática. Assim, este estudo justifica-se pela necessidade de se conhecer a ocorrência de violência no trabalho da Enfermagem na ESF a fim de oferecer uma contextualização sobre o campo de atuação da profissão e promover um maior debate e a sensibilização nos cenários investigados acerca da violência no trabalho.

Diante do exposto, definiram-se as seguintes questões norteadoras: "quais tipos de violência na ESF estão expostos os trabalhadores da equipe de Enfermagem e quais as influências dos aspectos laborais e do trabalhador?" O estudo objetivou analisar a ocorrência dos diferentes tipos de violência no trabalho da Enfermagem na ESF e as implicações dos aspectos laborais e do trabalhador.

\section{MÉTODO}

Estudo de métodos mistos, do tipo explanatório sequencial, em que foi conduzida uma etapa quantitativa (QUAN) seguida pela etapa qualitativa (QUAL), ${ }^{14}$ realizado com a ESF de um município localizado no Sul do Brasil. Na APS desse município, as Unidades Básicas de Saúde (UBS) são denominadas de Centros de Saúde da Família (CSF), formada por 26 CSF onde estão estruturadas 53 ESF, as quais compuseram o cenário de pesquisa.

Para a definição dos participantes, foram utilizados como critérios de inclusão: possuir formação na área da Enfermagem (enfermeiros, técnicos e auxiliares de Enfermagem) e estar atuando como profissional da ESF há, no mínimo, 12 meses, conforme requer o protocolo de pesquisa para o Survey. Foram excluídos os trabalhadores afastados por qualquer motivo no período da coleta de dados. Para a definição dos participantes da primeira etapa do estudo, foi realizado o cálculo amostral por categoria populacional elegível, considerando 95\% de confiança e erro de $5 \%$ da amostra. No período da coleta de dados, a população de trabalhadores constituía-se por 53 enfermeiros e 159 auxiliares/técnicos de Enfermagem. Assim, a amostra foi composta por 169 profissionais de Enfermagem, sendo 47 enfermeiros e 122 auxiliares/técnicos de Enfermagem. O estudo considerou auxiliares e técnicos de Enfermagem na mesma categoria por atuarem sem distinção de tarefas no cenário pesquisado. Dentre os trabalhadores que não fizeram parte da amostra, apenas uma auxiliar de Enfermagem recusou-se a participar da pesquisa e os demais não se enquadravam nos critérios de seleção estabelecidos. O período de coleta de dados ocorreu entre setembro de 2018 e março de 2019.

Na etapa QUAN da investigação, foram mensuradas as características demográficas e laborais dos trabalhadores e, para o levantamento da violência ocorrida nos últimos 12 meses, foi utilizado o Survey Questionnaire Workplace Violence in the Health Sector, proposto pela OMS, pela Organização Internacional do Trabalho e de Serviços Públicos e pelo Conselho Internacional de Enfermagem, ${ }^{15}$ traduzido e adaptado para a língua portuguesa. ${ }^{16}$ O questionário mensura a ocorrência dos tipos de violência física e psicológica, sendo a violência psicológica composta pela agressão verbal, pelo assédio moral, assédio sexual e pela discriminação racial.

Na etapa QUAL do estudo, foram convidados os profissionais que participaram da etapa anterior, sendo selecionados por 
sorteio, considerando a disponibilidade em participar de uma entrevista semiestruturada, incluindo dez trabalhadores que responderam ter sofrido diferentes tipos de violência no trabalho e oito trabalhadores que indicaram, em suas respostas, não ter sido expostos ao fenômeno, mas que sinalizaram ter observado violência contra os colegas no trabalho, totalizando 18 participantes. O quantitativo de participantes nessa etapa foi definido pela saturação de dados, ${ }^{17}$ sendo que as entrevistas foram gravadas e seguiram um roteiro semiestruturado. Ainda, essa etapa incluiu a observação não participante, as quais foram realizadas em $14 \mathrm{CSF}$, totalizando 56 horas de observação mediante um roteiro.

Para os registros de observações durante as coletas de dados, foi utilizado um Diário de Campo, identificado pelas iniciais (DC), o qual agregou informações para a completude das entrevistas, sendo registrados fatos, sentimentos, depoimentos e observações dos trabalhadores após cada etapa de coleta de dados. Os dados QUAN foram codificados, tabulados e analisados por meio do software Statistical Package for the Social Sciences, versão 21.0. As variáveis de natureza quantitativa foram descritas por meio de medidas de tendência central e dispersão: média; desvio-padrão; mediana; valores mínimo e máximo observados; amplitude interquartílica e a estimativa por intervalo de confiança para a média populacional com base no número de respostas válidas e nível de confiança de $95 \%$. O critério para a entrada da variável no modelo multivariado foi o valor $p<0,10$. As variáveis categóricas foram descritas por meio de frequências relativas e absolutas e as variáveis contínuas foram descritas com medidas de tendência central e dispersão. Após o teste de normalidade de Shapiro-Wilk, utilizou-se o teste qui-quadrado para a associação e MannWhitney para analisar as diferenças entre as medianas nos grupos.

Os dados provenientes das transcrições das entrevistas e trechos de observação foram submetidos à técnica de Análise Temática. ${ }^{18} \mathrm{~A}$ pré-análise dos achados foi configurada com base na leitura flutuante do material, identificação de categorias, subcategorias e unidades temáticas. Os QUAN e QUAL foram confrontados, buscando relações, convergências e divergências entre os resultados, o que permitiu o aprofundamento da análise de ocorrência da violência.

O estudo foi aprovado por um Comitê de Ética em Pesquisa, via Plataforma Brasil, sendo a aprovação sob o Parecer no 2.835.706/2018. Todos os participantes assinaram o Termo de Consentimento Livre e Esclarecido (TCLE). Para preservar o anonimato dos participantes no uso dos fragmentos das falas, estes foram codificados como: Enfermeiro $(E)$ ou Técnico de Enfermagem/Auxiliar de Enfermagem (TAE), seguido do número de ordem dos instrumentos.

\section{RESULTADOS}

Constituíram a amostra QUAN 169 trabalhadores que compõem as equipes de Enfermagem que atuam na ESF, sendo a maioria composta por mulheres (93,5\%), com idade média de 41,1 anos $( \pm 8,8)$, brancas $(n=154 / 91,1 \%)$, com companheiro $(n=119 / 70,4 \%)$ e que possuem, em média, um filho. As profissionais tinham, em média, 14 anos de experiência na área da saúde (mínimo de oito anos e máximo de 20 anos), com carga horária semanal de 40 horas, sendo que apenas $7,7 \%$ possuíam outro vínculo e 13\% ocupavam o cargo de gerentes do serviço, sendo que, majoritariamente, estabeleciam contato físico com o usuário $(94,1 \%)$.

No estudo, 141 trabalhadores revelaram ter sofrido, nos últimos 12 meses, 221 episódios de violência. Dentre eles, 76 $(45,0 \%)$ sofreram um tipo e 50 (29,6\%), dois tipos. A violência mais referida foi a psicológica, destacando-se a agressão verbal ( $n=128 / 75,7 \%)$, o assédio moral $(n=66 / 39,1 \%)$, o assédio sexual $(n=15 / 8,9 \%)$ e a discriminação racial $(n=7 / 4,1 \%)$. Ainda, parte da amostra $(n=5 / 3,0 \%)$ referiu ter sofrido violência física. No contexto, os usuários foram os principais perpetradores, exceto para o assédio moral.

A Tabela 1 apresenta as associações identificadas como significativas entre os diferentes tipos de violência contra os trabalhadores de Enfermagem da ESF e as variáveis de caracterização da amostra.

A Tabela 2 apresenta as associações da violência laboral com as características sociolaborais dos trabalhadores de Enfermagem.

A etapa QUAL do estudo contou com a participação de 18 trabalhadores, a qual permitiu melhor compreender o fenômeno da violência com os trabalhadores da ESF. Nessa etapa, respeitando o sequenciamento do estudo misto explanatório, foi possível identificar aspectos emergentes da ocorrência da violência no trabalho da Enfermagem na ESF, o que permite aprofundar alguns aspectos relacionados a alguns dados dos trabalhadores.

As entrevistas e observações registradas no DC possibilitaram expressar como a violência ocorre com os trabalhadores da ESF. Os resultados quantitativos mostram os enfermeiros mais expostos ao assédio moral e os técnicos/auxiliares de Enfermagem mais expostos ao assédio sexual e as entrevistas abaixo revelam as expressões dos trabalhadores vítimas.

Ele [colega médico] implicava com todas as minhas condutas no trabalho, chegava a instigar os pacientes a pensar que eu não sabia realizar consultas de Enfermagem adequadamente, que minhas orientações não serviam; isso se repetiu por mais de dez vezes no último ano. Um certo dia, me falou que iria me dar aulas para eu aprender a trabalhar... (E06)

No momento de observação, profissional enfermeiro se aproxima da pesquisadora e comenta que, durante o período eleitoral, o gestor instigou e pressionou os enfermeiros coordenadores a fazer campanha política e estar presente nos eventos políticos do município, destacou que sentia muito constrangimento porque a insistência persistia todas as semanas que antecederam as eleições. $O$ fato mais constrangedor e humilhante 
Tabela 1. Associações significativas entre os tipos de violência e os aspectos sociodemográficos e laborais dos profissionais de Enfermagem da ESF, Santa Catarina, 2020. ( $n=169)$

\begin{tabular}{|c|c|c|c|}
\hline \multicolumn{4}{|c|}{ AGRESSÃO FÍSICA } \\
\hline Variáveis & Sofreu Violência & Não Sofreu Violência & p-valor* \\
\hline Cor da pele & $n(\%)$ & $n(\%)$ & 0,625 \\
\hline Negra/Parda & $0(0,0 \%)$ & $15(100,0 \%)$ & \\
\hline Branca & $5(3,2 \%)$ & $149(96,8 \%)$ & \\
\hline Uso de medicamentos & n (\%) & n (\%) & 0,157 \\
\hline Sim & $4(4,7 \%)$ & $81(95,3 \%)$ & \\
\hline Não & $1(1,2 \%)$ & $83(98,8 \%)$ & \\
\hline Função & & & 0,057 \\
\hline Enfermeiro & $2(4,3 \%)$ & $45(95,7 \%)$ & \\
\hline Técnico/Auxiliar de Enfermagem & $3(2,4 \%)$ & $119(97,5 \%)$ & \\
\hline Estímulo para o relato de violência & & & 0,143 \\
\hline Sim & $2(7,7 \%)$ & $24(92,3 \%)$ & \\
\hline Não & $3(2,1 \%)$ & $140(97,9 \%)$ & \\
\hline \multicolumn{4}{|c|}{ AGRESSÃO VERBAL } \\
\hline Variáveis & Sofreu Violência & Não Sofreu Violência & p-valor* \\
\hline Cor da pele & $n(\%)$ & $n(\%)$ & 0,202 \\
\hline Negra/Parda & $13(86,6 \%)$ & $2(13,3 \%)$ & \\
\hline Branca & $115(74,7 \%)$ & $39(25,3 \%)$ & \\
\hline Uso de medicamentos & & & 0,037 \\
\hline Sim & $69(81,2 \%)$ & $16(18,8 \%)$ & \\
\hline Não & $59(70,2 \%)$ & $25(29,8 \%)$ & \\
\hline Função & & & 0,075 \\
\hline Enfermeiro & $36(76,6 \%)$ & $11(23,4 \%)$ & \\
\hline Técnico/Auxiliar de Enfermagem & $92(75,4 \%)$ & $30(24,5 \%)$ & \\
\hline Estímulo para o relato de violência & & & 0,110 \\
\hline $\operatorname{Sim}$ & $22(84,6 \%)$ & $4(15,4 \%)$ & \\
\hline Não & $106(74,1 \%)$ & $37(25,9 \%)$ & \\
\hline \multicolumn{4}{|c|}{ ASSÉDIO MORAL/INTIMIDAÇÃO } \\
\hline Variáveis & Sofreu Violência & Não Sofreu Violência & p-valor* \\
\hline Cor da pele & & & 0,047 \\
\hline Negra/Parda & $3(20,0 \%)$ & $12(80,0 \%)$ & \\
\hline Branca & $63(40,9 \%)$ & $91(59,1 \%)$ & \\
\hline Uso de medicamentos & & & 0,117 \\
\hline Sim & $32(37,6 \%)$ & $53(62,4 \%)$ & \\
\hline Não & $34(40,5 \%)$ & $50(59,5 \%)$ & \\
\hline Função & & & 0,010 \\
\hline Enfermeiro & $26(55,3 \%)$ & $21(44,7 \%)$ & \\
\hline Técnico/Auxiliar de Enfermagem & $40(32,7 \%)$ & $82(67,2 \%)$ & \\
\hline Estímulo para o relato de violência & & & 0,080 \\
\hline Sim & $13(50,0 \%)$ & $13(50,0 \%)$ & \\
\hline Não & $53(37,1 \%)$ & $90(62,9 \%)$ & \\
\hline
\end{tabular}

* p-valor obtido por meio do teste qui-quadrado de Pearson. Fonte: elaboração própria. 
Tabela 1. Continuação...

\begin{tabular}{|c|c|c|c|}
\hline \multicolumn{4}{|c|}{ ASSÉDIO SEXUAL } \\
\hline Variáveis & Sofreu Violência & Não Sofreu Violência & p-valor* \\
\hline Cor da pele & $\mathrm{n}(\%)$ & $n(\%)$ & 0,129 \\
\hline Negra/Parda & $3(20,0 \%)$ & $12(80,0 \%)$ & \\
\hline Branca & $12(7,8 \%)$ & $142(92,2 \%)$ & \\
\hline Uso de medicamentos & & & 0,206 \\
\hline $\operatorname{Sim}$ & $8(9,4 \%)$ & $77(90,6 \%)$ & \\
\hline Não & $7(8,3 \%)$ & $77(91,7 \%)$ & \\
\hline Função & & & 0,047 \\
\hline Enfermeiro & $2(4,3 \%)$ & $45(95,7 \%)$ & \\
\hline Técnico/Auxiliar de Enfermagem & $13(10,6 \%)$ & $109(89,3 \%)$ & \\
\hline Estímulo para o relato de violência & & & 0,044 \\
\hline Sim & $5(19,2 \%)$ & $21(80,8 \%)$ & \\
\hline Não & $10(7,0 \%)$ & $133(93,0 \%)$ & \\
\hline \multicolumn{4}{|c|}{ DISCRIMINAÇÃO RACIAL } \\
\hline Variáveis & Sofreu Violência & Não Sofreu Violência & p-valor* \\
\hline Cor da pele & $n(\%)$ & $n(\%)$ & 0,001 \\
\hline Negra/Parda & $4(26,5 \%)$ & $11(73,3 \%)$ & \\
\hline Branca & $3(1,9 \%)$ & $151(98,1 \%)$ & \\
\hline Uso de medicamentos & & & 0,166 \\
\hline Sim & $5(5,9 \%)$ & $80(94,1 \%)$ & \\
\hline Não & $2(2,4 \%)$ & $82(97,6 \%)$ & \\
\hline Função & & & 0,180 \\
\hline Enfermeiro & $1(2,1 \%)$ & $46(97,9 \%)$ & \\
\hline Técnico/Auxiliar de Enfermagem & $6(4,9 \%)$ & $116(95,0 \%)$ & \\
\hline Estímulo para o relato de violência & & & 0,303 \\
\hline Sim & $0(0,0 \%)$ & $26(100,0 \%)$ & \\
\hline Não & $7(4,9 \%)$ & $136(95,1 \%)$ & \\
\hline
\end{tabular}

* p-valor obtido por meio do teste qui-quadrado de Pearson. Fonte: elaboração própria.

ocorreu quando o gestor proferiu que entre um bom e competente enfermeiro e um bom enfermeiro político, sua preferência em manter no cargo de coordenação era pelo bom enfermeiro político...(DC)

Hoje de manhã, levei uma cantada de um paciente do sexo masculino... ele faz isso várias e várias e várias vezes e eu sempre tentando desconversar, mas parece que fico refém daquela situação, me sinto constrangido e coagido... Porque o que eu vou dizer, como que eu vou xingar ele, por exemplo, se eu arrumar uma inimizade, ele pode querer me agredir quando eu sair ali fora. (TAE105) [...] cada vez que ele [paciente idoso] vem pra unidade, fica se esfregando na gente, sempre dá um jeito de passar a mão, já levei tapinha na bunda, se aproxima para falar no ouvido, eu fico muito constrangida, não quero mais atender ele; além de abusado, não tem respeito com os profissionais. (TAE142)

Sobre os achados referentes à cor da pele e/ou etnia como aspecto associado à ocorrência de discriminação racial, apresentam-se registros da etapa QUAL.

A pesquisadora, ao realizar momento de observação em uma UBS, observa paciente do sexo masculino, idoso, proferir discriminação racial contra auxiliar de Enfermagem de etnia indígena. Se aproximou da recepção para 
Tabela 2. Associações entre a ocorrência dos diferentes tipos de violência e as características sociolaborais, Santa Catarina, 2020. $(n=169)$

\begin{tabular}{|c|c|c|c|c|}
\hline VIOLÊNCIA FISICA & & Média & Desvio-Padrão & p-valor* \\
\hline \multirow{2}{*}{ Escolaridade } & Sofreu violência & 15,5 & 3,122 & 0,780 \\
\hline & Não sofreu & 15,3 & 2,836 & \\
\hline \multirow{2}{*}{ Anos de experiência profissional } & Sofreu violência & 9,1 & 5,103 & 0,076 \\
\hline & Não sofreu & 15,3 & 8,630 & \\
\hline \multirow{2}{*}{ Tempo de serviço } & Sofreu violência & 5,4 & 3,460 & 0,495 \\
\hline & Não sofreu & 8,1 & 6,823 & \\
\hline \multirow{2}{*}{ Quantos profissionais atuam juntos } & Sofreu violência & 10,0 & 11,597 & 0,230 \\
\hline & Não sofreu & 6,1 & 9,241 & \\
\hline \multirow{2}{*}{ Satisfação local de trabalho } & Sofreu violência & 4,8 & 0,447 & 0,151 \\
\hline & Não sofreu & 4,3 & 0,699 & \\
\hline \multirow{2}{*}{ Reconhecimento no trabalho } & Sofreu violência & 4,4 & 0,547 & 0,177 \\
\hline & Não sofreu & 3,8 & 0,939 & \\
\hline \multirow{2}{*}{ Relacionamentos no trabalho } & Sofreu violência & 4,2 & 0,447 & 0,357 \\
\hline & Não sofreu & 4,3 & 0,632 & \\
\hline \multirow{2}{*}{ Preocupação com a violência } & Sofreu violência & 4,2 & 0,836 & 0,765 \\
\hline & Não sofreu & 3,9 & 1,120 & \\
\hline \multicolumn{2}{|l|}{ AGRESSÃO VERBAL } & Média & Desvio-Padrão & p-valor* \\
\hline \multirow{2}{*}{ Escolaridade } & Sofreu violência & 15,4 & 2,695 & 0,376 \\
\hline & Não sofreu & 14,9 & 3,237 & \\
\hline \multirow{2}{*}{ Anos de experiência profissional } & Sofreu violência & 14,4 & 7,789 & 0,234 \\
\hline & Não sofreu & 17,3 & 10,567 & \\
\hline \multirow{2}{*}{ Tempo de serviço } & Sofreu violência & 7,8 & 6,802 & 0,502 \\
\hline & Não sofreu & 8,6 & 6,668 & \\
\hline \multirow{2}{*}{ Quantos profissionais atuam juntos } & Sofreu violência & 5,8 & 8,777 & 0,056 \\
\hline & Não sofreu & 7,4 & 10,807 & \\
\hline \multirow{2}{*}{ Satisfação local de trabalho } & Sofreu violência & 4,3 & 0,698 & 0,058 \\
\hline & Não sofreu & 4,3 & 0,674 & \\
\hline \multirow{2}{*}{ Reconhecimento no trabalho } & Sofreu violência & 3,7 & 0,955 & 0,010 \\
\hline & Não sofreu & 4,1 & 0,781 & \\
\hline \multirow{2}{*}{ Relacionamentos no trabalho } & Sofreu violência & 4,3 & 0,641 & 0,022 \\
\hline & Não sofreu & 4,5 & 0,540 & \\
\hline \multirow{2}{*}{ Preocupação com a violência } & Sofreu violência & 4,0 & 1,005 & 0,098 \\
\hline & Não sofreu & 3,6 & 1,355 & \\
\hline
\end{tabular}

* p-valor obtidos por meio do teste Mann-Whitney, teste que compara a diferença de medianas devido à assimetria das variáveis. No entanto, para facilitar a interpretação dos achados, optou-se por apresentar as médias e o desvio-padrão. Fonte: elaboração própria. 
Tabela 2. Continuação...

\begin{tabular}{|c|c|c|c|c|}
\hline ASSÉDIO MORAL/INTIMIDAÇÃO & & Média & Desvio-Padrão & p-valor* \\
\hline \multirow{2}{*}{ Escolaridade } & Sofreu violência & 15,6 & 3,073 & 0,151 \\
\hline & Não sofreu & 15,1 & 2,666 & \\
\hline \multirow{2}{*}{ Anos experiência profissional } & Sofreu violência & 14,1 & 8,589 & 0,151 \\
\hline & Não sofreu & 15,8 & 8,594 & \\
\hline \multirow{2}{*}{ Tempo de serviço } & Sofreu violência & 7,1 & 5,605 & 0,393 \\
\hline & Não sofreu & 8,6 & 7,368 & \\
\hline \multirow{2}{*}{ Quantidade de profissionais com quem atua } & Sofreu violência & 6,7 & 9,648 & 0,776 \\
\hline & Não sofreu & 5,9 & 9,104 & \\
\hline \multirow{2}{*}{ Satisfação local de trabalho } & Sofreu violência & 4,2 & 0,729 & 0,088 \\
\hline & Não sofreu & 4,4 & 0,667 & \\
\hline \multirow{2}{*}{ Reconhecimento no trabalho } & Sofreu violência & 3,6 & 1,021 & 0,013 \\
\hline & Não sofreu & 4,0 & 0,839 & \\
\hline \multirow{2}{*}{ Relacionamento no trabalho } & Sofreu violência & 4,2 & 0,651 & 0,007 \\
\hline & Não sofreu & 4,4 & 0,592 & \\
\hline \multirow{2}{*}{ Preocupação com a violência } & Sofreu violência & 4,2 & 1,023 & 0,004 \\
\hline & Não sofreu & 3,7 & 1,134 & \\
\hline \multicolumn{2}{|l|}{ ASSÉDIO SEXUAL } & Média & Desvio-Padrão & p-valor* \\
\hline \multirow{2}{*}{ Escolaridade } & Sofreu violência & 14,6 & 2,065 & 0,183 \\
\hline & Não sofreu & 15,4 & 2,894 & \\
\hline \multirow{2}{*}{ Anos experiência profissional } & Sofreu violência & 13,2 & 7,816 & 0,325 \\
\hline & Não sofreu & 15,3 & 8,676 & \\
\hline \multirow{2}{*}{ Tempo de serviço } & Sofreu violência & 6,9 & 5,463 & 0,619 \\
\hline & Não sofreu & 8,1 & 6,876 & \\
\hline \multirow{2}{*}{ Quantidade de profissionais com quem atua } & Sofreu violência & 3,4 & 3,720 & 0,243 \\
\hline & Não sofreu & 6,5 & 9,638 & \\
\hline \multirow{2}{*}{ Satisfação local de trabalho } & Sofreu violência & 4,2 & 0,703 & 0,482 \\
\hline & Não sofreu & 4,3 & 0,697 & \\
\hline \multirow{2}{*}{ Reconhecimento no trabalho } & Sofreu violência & 3,8 & 1,014 & 0,852 \\
\hline & Não sofreu & 3,8 & 0,929 & \\
\hline \multirow{2}{*}{ Relações de trabalho } & Sofreu violência & 4,3 & 0,617 & 0,649 \\
\hline & Não sofreu & 4,3 & 0,630 & \\
\hline \multirow{2}{*}{ Preocupação com a violência } & Sofreu violência & 4,4 & 0,828 & 0,113 \\
\hline & Não sofreu & 3,9 & 1,129 & \\
\hline
\end{tabular}

* p-valor obtidos por meio do teste Mann-Whitney, teste que compara a diferença de medianas devido à assimetria das variáveis. No entanto, para facilitar a interpretação dos achados, optou-se por apresentar as médias e o desvio-padrão. Fonte: elaboração própria. 
Tabela 2. Continuação...

\begin{tabular}{|c|c|c|c|c|}
\hline DISCRIMINAÇÃO RACIAL & & Média & Desvio-Padrão & p-valor* \\
\hline \multirow{2}{*}{ Escolaridade } & Sofreu violência & 14,5 & 1,766 & 0,312 \\
\hline & Não sofreu & 15,4 & 2,871 & \\
\hline \multirow{2}{*}{ Anos experiência profissional } & Sofreu violência & 14,2 & 9,282 & 0,797 \\
\hline & Não sofreu & 15,2 & 8,601 & \\
\hline \multirow{2}{*}{ Tempo de serviço } & Sofreu violência & 4,9 & 4,686 & 0,264 \\
\hline & Não sofreu & 8,2 & 6,812 & \\
\hline \multirow{2}{*}{ Quantidade de profissionais com quem atua } & Sofreu violência & 3,5 & 2,878 & 0,812 \\
\hline & Não sofreu & 6,3 & 9,467 & \\
\hline \multirow{2}{*}{ Satisfação local de trabalho } & Sofreu violência & 4,8 & 0,378 & 0,049 \\
\hline & Não sofreu & 4,3 & 0,700 & \\
\hline \multirow{2}{*}{ Reconhecimento no trabalho } & Sofreu violência & 3,0 & 1,397 & 0,369 \\
\hline & Não sofreu & 3,8 & 0,910 & \\
\hline \multirow{2}{*}{ Relações de trabalho } & Sofreu violência & 4,5 & 0,534 & 0,471 \\
\hline & Não sofreu & 4,3 & 1,127 & \\
\hline \multirow{2}{*}{ Preocupação com a violência } & Sofreu violência & 14,5 & 0,534 & 0,345 \\
\hline & Não sofreu & 15,4 & 1,127 & \\
\hline
\end{tabular}

* p-valor obtidos por meio do teste Mann-Whitney, teste que compara a diferença de medianas devido à assimetria das variáveis. No entanto, para facilitar a interpretação dos achados, optou-se por apresentar as médias e o desvio-padrão. Fonte: elaboração própria.

solicitar atendimento e referiu não querer ser atendido pelo profissional, denominando-o por "bugre". (DC)

O paciente chegou na unidade e, quando me viu, pediu para não ser atendido pelo "bugre". (TAE87)

Durante a manhã de observações, enfermeira se aproxima da pesquisadora e relata que auxiliar de Enfermagem negra sofreu discriminação racial de paciente por estar como calçado sujo de lama em um dia que choveu muito. Ela se sentiu muito humilhada e atribuiu isso à sua cor de pele... a partir deste dia, ela troca o calçado ao entrar na UBS. (DC)

Na etapa QUAL, alguns profissionais também revelaram que têm mais satisfação em atuar nesse local de trabalho, tendo em vista que se sentem menos expostos à violência, o que proporciona a sensação de segurança.

Após o término de um atendimento, em que se observa a cordialidade e respeito entre a profissional de Enfermagem e o usuário em atendimento, a profissional, ao finalizar, verbaliza sobre a atuação de oito anos na unidade, menciona que gosta do que faz e sobre a satisfação que o atendimento do usuário Ihe proporciona e sobre sentir-se segura naquele local de atuação. (DC)

\section{DISCUSSÃO}

O estudo evidenciou a elevada incidência $(83,4 \%)$ de violência perpetrada contra os trabalhadores de Enfermagem na ESF e este achado reforça o encontrado em outros estudos com trabalhadores da APS, ${ }^{11,19} \mathrm{com}$ resultados consonantes ${ }^{20}$ e dissonantes ${ }^{21}$ de outras pesquisas que utilizaram o mesmo inquérito para a investigação. Já em outra investigação, realizada na região Sul do Brasil, ${ }^{9}$ a prevalência da violência foi de $69,8 \%$ de trabalhadores e, em Minas Gerais, 76,8\% dos trabalhadores relataram ter sofrido vários episódios de agressões no trabalho. ${ }^{22}$

A violência psicológica foi a mais prevalente evidenciada neste estudo, destacando-se, dentre os seus subtipos, a agressão verbal praticada contra os profissionais de saúde, confirmando

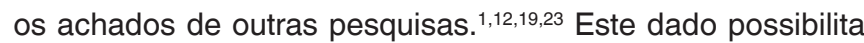
dizer que a prevalência da agressão verbal pode indicar falhas na comunicação entre os usuários e os profissionais da área da saúde. ${ }^{12}$

No que concerne à identificação do principal agressor em atos contra os trabalhadores de Enfermagem da ESF, revelaramse achados semelhantes aos de outras pesquisas, ${ }^{1,10,12}$ que evidenciaram os usuários dos serviços como os principais perpetradores. O comportamento desses, ao desrespeitarem os trabalhadores, gera insatisfação profissional, fato que pode ser atrelado, algumas vezes, ao não entendimento dos usuários quanto aos preceitos estabelecidos no modelo de atenção 
da $\mathrm{APS}^{24}$ e por sentirem que não receberam um tratamento adequado. ${ }^{12}$ Frequentemente, a falta de resolutividade desse nível assistencial também pode representar um fator contribuinte para desencadear agressões e ameaças no ambiente laboral pelos usuários. ${ }^{12}$

A violência física revelou uma associação estatística com a satisfação pelo local de trabalho, comprovando que os trabalhadores mais satisfeitos sofreram menos violência física. Não sofrer o fenômeno tem impacto positivo na satisfação laboral, no sentimento de reconhecimento e nos relacionamentos interpessoais. Os profissionais que atuam no modelo ESF relatam que os motivos que geram satisfação no local de trabalho estão relacionados ao comprometimento da equipe, salário favorável, prática de acolhimento, vínculo com usuários, autonomia para a realização do trabalho, resolubilidade da assistência, afinidade com a profissão e gosto pelo que fazem. ${ }^{24}$

Identificou-se que os trabalhadores que sofreram agressão verbal apresentaram menores médias na avaliação sobre o reconhecimento e os relacionamentos no trabalho. Outra investigação verificou que ter o relacionamento interpessoal desfavorável foi um fator significativo para o desfecho da violência entre os trabalhadores e a maioria das vítimas de agressões revelou-se preocupada com o fenômeno no local de trabalho. ${ }^{25}$ Também, a falta de reconhecimento no trabalho, externada por falta de respeito e abuso verbal, provoca adoecimento, sofrimento e processos de despersonalização dos trabalhadores. ${ }^{26}$ Além da influência no desempenho do trabalhador, as consequências da violência no trabalho perpassam a instituição de saúde, com repercussões importantes nas dimensões familiar e social dos profissionais de Enfermagem. ${ }^{27}$

Ainda se constatou que a distribuição de trabalhadores que usam medicamentos é maior entre os expostos à agressão verbal. Outro dado encontrado foi que $50,3 \%$ dos trabalhadores utilizam medicamentos para o alívio da depressão, da ansiedade, do aumento dos níveis de hipertensão arterial, entre outras patologias. Um estudo com trabalhadores da APS mencionou que esses enfrentam dores e queixas físicas relacionadas ao estresse do ambiente laboral, caracterizado por conflitos, falta de reconhecimento profissional, sobrecarga de trabalho e falta de recursos. ${ }^{28}$

No que diz respeito ao assédio moral, os resultados indicaram que os participantes de raça branca e os enfermeiros têm maior propensão de sofrer assédio moral. Este fator pode estar relacionado aos trabalhadores dessa categoria estarem acostumados com a intimidação nos serviços, contudo, essa repercute em avaliações mais negativas acerca do reconhecimento e dos relacionamentos no trabalho, bem como uma maior preocupação com a violência nesse contexto. A intimidação, ao trabalhador, tem causado adoecimento, com repercussão negativa nas suas funções laborais, sendo que essa tem sido considerada aceita e reproduzida diante da cultura organizacional das instituições de saúde. ${ }^{29}$ No cenário internacional, ${ }^{30}$ identificou-se que os profissionais de Enfermagem mais expostos a essa violência também manifestaram sentimento de pouco reconhecimento $e$ confiança no trabalho que realizam. $O$ assédio moral repercute na saúde do trabalhador, desestabiliza o equilíbrio físico e emocional, deixando marcas permanentes na vida pessoal e laboral das vítimas. ${ }^{31}$ Estudos comprovaram que, independentemente das características dos enfermeiros, bem como da frequência com que ocorre, o assédio moral tem impacto negativo na saúde mental e no bem-estar dos enfermeiros. ${ }^{32}$

Evidenciou-se que $o$ assédio sexual foi mais comum e mais repetido entre os técnicos/auxiliares de Enfermagem. Sobre esse tipo de violência, também se identificou a dificuldade de os trabalhadores relatarem os episódios por sentirem medo e vergonha..$^{33}$

Constatou-se que perceber que não existe estímulo para o relato da violência foi associado ao assédio sexual e que, de maneira geral, os profissionais não identificam esse recurso no contexto do serviço. $O$ estímulo ao relato dessas situações pode ser conduzido por meio da comunicação eficaz e deve primar pela escuta como estratégia de gerenciamento da violência. ${ }^{24}$ Nesse sentido, propõem-se formas de contenção a todos os tipos de violências contra os profissionais de Enfermagem, assim como a elaboração de medidas para a proteção das vítimas, considerando-se os aspectos que se associam a cada tipo.

$A$ análise da discriminação racial revelou que ser negro $e$ pardo tem maior propensão a sofrer discriminação racial e os trechos de falas e observações robustecem esses achados, apesar do número reduzido desses na amostra pesquisada, o que pode sinalizar para a magnitude desse problema. $O$ Conselho Regional de Enfermagem, em um estudo sobre o perfil da Enfermagem no Brasil, apresentou uma realidade traçada por marcas históricas, caracterizada por desigualdades raciais e sociais, em que as pessoas negras foram excluídas da profissionalização na categoria e as que atuam sofrem episódios de preconceito. $\mathrm{O}$ racismo mostrou-se velado e naturalizado, evidenciado nas recusas dos pacientes de atendimento por profissionais negros e no sentimento de falta de reconhecimento profissional. ${ }^{34}$

Dos resultados do estudo, emerge a necessidade de se repensarem as estratégias de intervenção com vistas a prevenir e/ou mitigar a ocorrência de violência no trabalho. Alguns modelos preconizam a existência de estratégias que incluam a abordagem em diversos níveis: individual, relacional e organizacional. As estratégias em nível individual estão relacionadas com as crenças, as atitudes e os comportamentos de cada pessoa; as estratégias relacionais têm enfoque na forma como as pessoas se relacionam e comunicam com os outros e, por fim, as estratégias organizacionais são centradas no clima e na estrutura organizacional, bem como nas políticas e nos procedimentos. ${ }^{35}$ Os autores confirmam que as estratégias com maior potencial de eficácia são as organizacionais, essencialmente centradas em ambientes de trabalho saudáveis, que impeçam a existência de qualquer um dos tipos de violência. ${ }^{35}$

Os resultados QUAN e QUAL mostram-se complementares, fortalecendo a análise dos dados e favorecendo a compreensão 
desse fenômeno complexo que ocorre no cenário da ESF envolvendo a Enfermagem.

\section{CONCLUSÃO E IMPLICAÇÕES PARA A PRÁTICA}

O estudo revelou que os trabalhadores de Enfermagem da ESF estão expostos à violência em seu ambiente. Comprovouse que a agressão verbal, marcadamente ilustrada nas falas e observações, tem implicações entre os profissionais em menores médias na avaliação sobre o reconhecimento e os relacionamentos no trabalho, bem como o uso de medicamentos é mais prevalente entre os expostos a esse tipo de agressão.

$O$ assédio moral foi influenciado pela cor da pele branca, entre enfermeiros, e repercute em médias mais baixas na avaliação do reconhecimento e relacionamentos no trabalho e maior preocupação com a violência no trabalho com maior dificuldade de relatos sobre o fenômeno. Já o assédio sexual foi significativo entre os técnicos/auxiliares de Enfermagem, influenciado pela ausência de estímulo para os relatos de violência. Por fim, a discriminação racial associou-se à cor da pele negra/ parda, apresentando uma redução da satisfação com o local de trabalho, sendo todos os tipos de assédio mais difíceis de serem observados e relatados pelos profissionais, que discorrem sobre o medo e a vergonha em denunciar os episódios.

Os resultados deste estudo apontam para a necessidade de planejamento de medidas contra a violência e de diretrizes que viabilizem o trabalho seguro e acolhedor aos trabalhadores, fator primordial para a manutenção da saúde do trabalhador, assim como para a qualidade da assistência prestada aos usuários.

A análise individualizada dos diferentes tipos de violência no trabalho da Enfermagem na ESF e suas associações com as características sociodemográficas e laborais desses profissionais indicam condutas específicas para cada fenômeno e reforçam a importância do relato e das denúncias dos episódios para a gestão dos serviços de saúde.

$O$ estudo limitou-se a investigar a violência somente com trabalhadores de Enfermagem que atuam na ESF, contudo, os demais trabalhadores da área da saúde também podem estar expostos ao fenômeno. Recomenda-se que outras pesquisas sejam realizadas considerando-se a ampliação dos participantes e as abordagens.

\section{FINANCIAMENTO}

Este trabalho foi realizado com o apoio da Coordenação de Aperfeiçoamento de Pessoal de Nível Superior - Brasil (CAPES) - Código de Financiamento 001; bolsa de doutorado concedida a Grasiele Fatima Busnello. Fundação de Amparo à Pesquisa de Santa Catarina (FAPESC) (Financiamento de Pesquisa Edital PAP/FAPESC TR1153), coordenado por: Letícia de Lima Trindade.

\section{CONTRIBUIÇÕES DOS AUTORES}

Desenho do estudo. Grasiele Fatima Busnello.

Coleta ou produção dos dados. Grasiele Fatima Busnello.

Letícia de Lima Trindade. Daiane Dal Pai.

Análise de dados. Grasiele Fatima Busnello. Carmem Lúcia

Colomé Beck. Letícia de Lima Trindade. Daiane Dal Pai.

Interpretação dos resultados. Grasiele Fatima Busnello.

Olga Maria Pimenta Lopes Ribeiro. Carmem Lúcia Colomé Beck.

Letícia de Lima Trindade. Daiane Dal Pai.

Redação e revisão crítica do manuscrito. Grasiele Fatima Busnello. Letícia de Lima Trindade. Daiane Dal Pai. Carmem Lúcia Colomé Beck. Olga Maria Pimenta Lopes Ribeiro.

Aprovação da versão final do artigo. Grasiele Fatima Busnello. Letícia de Lima Trindade. Daiane Dal Pai. Carmem Lúcia Colomé Beck. Olga Maria Pimenta Lopes Ribeiro.

Responsabilidade por todos os aspectos do conteúdo e pela integridade do artigo publicado. Grasiele Fatima Busnello. Letícia de Lima Trindade. Daiane Dal Pai. Carmem Lúcia Colomé Beck. Olga Maria Pimenta Lopes Ribeiro.

\section{EDITOR ASSOCIADO}

\author{
Gerson Luiz Marinho
}

\section{REFERÊNCIAS}

1. Dal Pai D, Sturbelle ICS, Santos C, Tavares JP, Lautert L. Violência física e psicológica perpetrada no trabalho em saúde. Texto Contexto Enferm. 2018;27(1):e2420016. http://dx.doi.org/10.1590/010407072018002420016.

2. Organização Mundial da Saúde. Relatório Mundial Sobre Violência e Saúde. Brasília: OMS/OPAS; 2002.

3. Organización Internacional del Trabajo, Consejo Internacional de Enfermeras, Organización Mundial de laSalud/Internacional de Servicios Públicos. Directrices marco para afrontar la violencia laboral en el sector de la salud. Ginebra: Organización Internacional del Trabajo; 2002.

4. Vincent-Höper S, Stein M, Nienhaus A, Schablon A. Workplace aggression and burnout in nursing: the moderating role of follow-up counseling. int jenviron res public health. 2020;17(9):3152. http://dx.doi.org/10.3390/ ijerph17093152. PMid:32369903.

5. Pompeii LA, Schoenfisch AL, Lipscomb HJ, Dement JM, Smith CD, Upadhyaya M. Physical assault, physical threat, and verbal abuse perpetrated against hospital workers by patients or visitors in six US hospitals. Am J Ind Med. 2015;58(11):1194-204. http://dx.doi.org/10.1002/ ajim.22489. PMid:26076187.

6. Abdellah RF, Salama KM. Prevalence and risk factors of workplace violence against health care workers in emergency department in Ismailia, Egypt. Pan Afr Med J.2017;26(21):21. http://dx.doi.org/10.11604/ pamj.2017.26.21.10837. PMid:28451000.

7. Schablon A, Wendeler D, Kozak A, Nienhaus A, Steinke S. Prevalence and consequences of aggression and violence towards nursing and care staff in germany: a survey. Int J Environ Res Public Health. 2018;15(6):1274. http://dx.doi.org/10.3390/ijerph15061274. PMid:29914142.

8. Ruiz-Hernández JA, López-García C, Llor-Esteban B, Galián-Muñoz I, Benavente-Reche AP. Evaluation of the users violence in primary health care: adaptation of an instrument. Int $\mathrm{J}$ Clin Health Psychol. 2016;16(3):295-305. http://dx.doi.org/10.1007/s00127-015-1039-9. PMid:30487873.

9. Fisekovic Kremic MB, Terzic-Supic ZJ, Santric-Milicevic MM, Trajkovic GZ. Encouraging employees to report verbal violence in primary health care in Serbia: a cross-sectional study. Zdr Varst. 2016;56(1):11-7. http:// dx.doi.org/10.1515/sjph-2017-0002. PMid:28289458. 
10. Ministério da Saúde (BR). Política Nacional de Atenção Básica. Brasília: Ministério da Saúde; 2017.

11. Silva ATC, Peres MFT, Lopes CS, Schraiber LB, Susser E, Menezes PR. Violence at work and depressive symptoms in primary health care teams: a cross-sectional study in Brazil. Soc Psychiatry Psychiatr Epidemiol. 2015;50(9):1347-55. http://dx.doi.org/10.1007/s00127-015-1039-9. PMid:25777684.

12. Sturbelle ICS, Dal Pai D, Tavares JP, Trindade LL, Riquinho DL, Ampos LF. Violência no trabalho em saúde da família: estudo de métodos mistos. Acta Paul Enferm. 2019;32(6):632-41. http://dx.doi.org/10.1590/19820194201900088

13. Flórido HG, Duarte SCM, Floresta WMC, Marins AMF, Broca PV, Moraes JRMM. Gerenciamento das situações de violência no trabalho na estratégia de saúde da família pelo enfermeiro. Texto Contexto Enferm. 2020;29:e20180432.

14. Creswell JW, Clark VLP. Pesquisa de métodos mistos. 2. ed. Porto Alegre: Penso; 2013.

15. Di Martino V. Relationship between work stress and workplace violence in the health sector. Geneva: World Health Organization; 2003.

16. Palácios $M$. Relatório preliminar de pesquisa, violência no trabalho no setor saúde - Rio de Janeiro - Brasil [Internet]. Rio de Janeiro: Universidade Federal do Rio de Janeiro; 2002 [citado 2020 mar 20]. Disponível em: http://www.assediomoral.org/IMG/pdf/pesquisa_sobre_ Violencia_no_trabalho_Universidade_Federal_RJ.pdf

17. Minayo MCS. Amostragem e saturação em pesquisa qualitativa: consensos e controvérsias. Rev Pesquisa Qualitativa [Internet]. 2017; [citado 2020 mar 20];5(7):1-12. Disponível em. http://rpq.revista.sepq. org.br/index.php/rpq/article/view/82

18. Bardin L. Análise de conteúdo. Ed. rev e ampl. São Paulo: Editora 70 2016. 279 p.

19. Contrera L, Monteiro I. Violência e capacidade para o trabalho entre trabalhadores de enfermagem brasileiros. São Paulo: Unicamp BFCM; 2017.

20. Trindade LL, Ribeiro ST, Zanatta EA, Vendruscolo C, Dal Pai D. Agressão verbal no trabalho da Enfermagem na área hospitalar. Rev Eletron Enferm. 2019;21(54333):1-8. http://dx. doi.org/10.5216/ree.v21.54333.

21. Cheung T, Lee PH, Yip PSF. Workplace Violence toward Physicians and Nurses: Prevalence and Correlates in Macau. Int J Environ Res Public Health. 2017;14(8):879. http://dx.doi.org/10.3390/ijerph14080879. PMid:28777333.

22. Vieira GLC. Agressão física contra técnicos de enfermagem em hospitais psiquiátricos. Rev Bras Saúde Ocup. 2017;42:e8. http://dx.doi. org/10.1590/2317-6369000004216.

23. Tsukamoto SAS, Galdino MJQ, Robazzi MLCC, Ribeiro RP, Soares MH, Haddad MCFL et al. Violência ocupacional na equipe de enfermagem: prevalência e fatores associados. Acta Paul Enferm. 2019;32(4):425-32. http://dx.doi.org/10.1590/1982-0194201900058.
24. Soratto J, Pires DEP, Trindade LL, Oliveira JSMA, Forte ECN, Melo TP Insatisfacão no trabalho de profissionais da saúde na Estratégia Saúde da Família. Texto Contexto Enferm. 2017;26(3):e2500016. http://dx.doi. org/10.1590/0104-07072017002500016.

25. Lima DM, Santos DF, Oliveira FN, Fonseca APLA, Passos JP.Violencia psicológica institucional no trabalho da enfermagem. Rev Pesq Cuid Fundam. 2012;4(1):17-20. http://dx.doi.org/10.9789/2175-5361.rpcfo. v0.1651.

26. Silva RVS, Deusdedit-Júnior M, Batista MA. A relação entre reconhecimento, trabalho e saúde sob o olhar da Psicodinâmica do Trabalho e da Clínica da Atividade: debates em psicologia do trabalho. Gerais Rev Interins Psicol [Internet]. 2015; [citado 2020 mar 20];8(2):415-27. Disponível em: http://pepsic.bvsalud.org/scielo.php?script=sci_arttext\&pid=S1983$82202015000300010 \&$ lng $=p t$

27. Bordignon M, Monteiro MI. Violence in the workplace in Nursing consequences overview. Rev Bras Enferm. 2016;69(5):996-9. http:// dx.doi.org/10.1590/0034-7167-2015-0133. PMid:27783746.

28. Dashtipour P, Vidaillet B. Work as affective experience: the contribution of Christophe Dejours' 'psychodynamics of work'. Organization. 2017;24(1):18-35. http://dx.doi.org/10.1177/1350508416668191.

29. Vasconcellos IRR, Griep RH, Lisboa MTL, Rotenberg L. Violence in daily hospital nursing work. Acta Paul Enferm. 2012;25(spe2):40-7. http://dx.doi.org/10.1590/S0103-21002012000900007.

30. Wu S, Zhu W, Li H, Lin S, Chai W, Wang X. Workplace violence and influencing factors among medical professionals in China. Am J Ind Med. 2012;55(11):1000-8. http://dx.doi.org/10.1002/ajim.22097. PMid:22886819.

31. Andrade CG, Leão JDM, Costa ICP, Brito FM, Santos KFO, Costa SFG. Assédio moral na atenção básica segundo os profissionais de enfermagem. Trab Educ Saúde. 2015;13(Supl. 1):77-90. http://dx.doi. org/10.1590/1981-7746-sip00031.

32. Harb A, Al Rayan A, Al.khashashneh OZ. The relationship between workplace bullying and positive mental health among registered nurses. J Am Psychiatr Nurses Assoc. 2019. http://dx.doi org/10.1177/1078390319877223.

33. Douglas KE, Nkporbu AK. Prevalence and pattern of workplace violence and ethnic discrimination among workers in a tertiary institution in Southern Nigeria. OALib J. 2017;4(3):3464. http://dx.doi.org/10.4236/ oalib.1103464.

34. Conselho Federal de Enfermagem. Mulheres e negros são maioria entre os profissionais de Enfermagem em MT [Internet]. 2018 [citado 2020 mar 20]. Disponível em: http://www.cofen.gov.br/mulheres-e-negrossao-maioria-entre-os-profi-em-mt_66743.htmlssionais-de-enfermagem

35. Smith CR, Palazzo SJ, Grubb PL, Gillespie GL. Standing up against workplace bullying behavior: recommendations from newly licensed nurses. J Nurs Educ Pract. 2020;10(7):1-2. 